\title{
Behavior of biodegradable oil under impulse voltages
}

\begin{abstract}
The properties of Palm Oil (PO) and Coconut Oil (CO) offer the potential for transformers with non-toxicity, high fire and flash points and better environmental compatibility while compared with those filled with Mineral Oil (MO). This potential has led to intensive studies of electrical performance of biodegradable oil especially in evaluating the electrical performance under lightning impulse voltage in recent years. This paper presents the investigation on the impulse breakdown voltage of $\mathrm{PO}$ and $\mathrm{CO}$ in such a uniform field. The PO used in this study is Refined, Bleached and Deodorized Palm Oil (RBDPO) Olein type. Two testing methods, rising-voltage and up-and-down are considered for both oils with different gap distances $(2.0 \mathrm{~mm}$ and $3.8 \mathrm{~mm})$. Testing methods including rising-voltage method and up-and-down method have no notable influence on the breakdown voltages of RBDPOs and CO compared to MO.IntroductionThe majority of transformers in the power system network are oil filled type. The main functions of the oil used in a transformer are to act as electrical insulation, cooling medium and information carrier. Up to now, the main oil insulation used in a transformer is Mineral Oil (MO) since it is proven to be successfully applied in practice for many years. Natural and synthetic esters are considered as the alternative for conventional MO due to the high fire safety and environmental friendliness. Extensive studies were conducted looking into the chemical, physical and electrical properties of natural and synthetic esters [1-3]. Another possible alternative for ester based oils are Palm Oil (PO) and Coconut Oil (CO). PO is derived from palm kernel which can be further separated to produce Crude Palm Oil (CPO) and Crude Palm Kernel Oil (CPKO) [45]. Further processing of CPO can produced Refined, Bleaching and Deodorized Palm Oil (RBDPO). The most common PO considered for transformers application is RBDPO. On the other hand, $\mathrm{CO}$ is extracted from kernel of matured coconut palm. The $\mathrm{CO}$ can be further processed to produce a neutralized, bleached and deodorized $\mathrm{CO}$ and is currently the type of oil investigated for the transformers' application [6]. Transformer in service shall be exposed to voltages in excess of the normal operating voltage, such as transient overvoltage due to lightning impulse or switching impulse. Therefore the increasing interests among researchers press for comprehensive investigations on electrical performance of these liquids especially under impulse voltage [7]. This paper presents breakdown strengths of biodegradable oils (RBDPOs and CO) and MO in a uniform sphere-sphere electrode under impulse voltages. The influences of voltage polarities (positive and negative) and testing methods (risingvoltage and up-and-down) on the breakdown behavior are investigated.
\end{abstract}

Keyword: Transformers oil; Impulse breakdown strength; Uniform field; Palm oil, Coconut oil 